Applied Mathematical Sciences, Vol. 7, 2013, no. 24, 1199 - 1203

HIKARI Ltd, www.m-hikari.com

\title{
The Identity Checking Problem \\ for Semigroups
}

\author{
Anna Gorbenko \\ Department of Intelligent Systems and Robotics \\ Ural Federal University \\ 620083 Ekaterinburg, Russia \\ gorbenko.ann@gmail.com \\ Vladimir Popov \\ Department of Intelligent Systems and Robotics \\ Ural Federal University \\ 620083 Ekaterinburg, Russia \\ Vladimir.Popov@usu.ru
}

\begin{abstract}
In this paper, we consider the identity checking problem for semigroups. We propose a genetic algorithm to solve the problem.
\end{abstract}

Keywords: identity checking problem, semigroup, genetic algorithm

There is a considerable interest in investigation of semigroup identities (see e.g. [1] - [4]). In particular, the identity checking problem for finite semigroups is extensively studied (see e.g. [5] and references in [5]). The identity checking problem in semigroup $\mathcal{A}$ is the following combinatorial decision problem.

CHECK-ID $(\mathcal{A})$ :

INSTANCE: Words of variables $u$ and $v$.

QUESTION: Whether or not the identity $u=v$ holds in $\mathcal{A}$ ?

Usually, a semigroup for an instance of the identity checking problem for finite semigroups is given by a semigroup multiplication table. There is a finite semigroup $\mathcal{A}$ such that CHECK-ID $(\mathcal{A})$ is co-NP-complete (see e.g. [5]).

Note that a large number of algorithmic problems of robotics received a lot of attention recently (see e.g. [6] - [15]). The representation of robotic systems plays an important role in solutions of robotic tasks (see e.g. [16]). There is a natural way to represent a robotic system by elements of some semigroup (see e.g. [17]). But in this case, we need to consider exponentially 
large or infinite semigroups. Therefore, for robotic systems, a representation by semigroup relations is preferred. It should be noted that in some cases a representation by relations and identities may be considerably shorter than any representation by relations. So, for robotic systems, it is intresting to consider the problem $\operatorname{CHECK}-\operatorname{ID}(\mathcal{A})$ where $\mathcal{A}$ is a description of some robotic system. In this paper, we assume that a semigroup is given by a set of semigroup relations. In this case, also there is a finite semigroup $\mathcal{A}$ such that $\operatorname{CHECK}-\operatorname{ID}(\mathcal{A})$ is coNP-complete. There is a infinite semigroup $\mathcal{A}$ such that $\operatorname{CHECK}-\operatorname{ID}(\mathcal{A})$ is undecidable (see e.g. [18]). In this paper, we consider a genetic algorithm to solve the problem.

Let $\Sigma$ be a finite system of semigroup relations. Let

$$
\mathcal{A}=\langle a[1], \ldots, a[n] \mid \Sigma\rangle
$$

be a semigroup where $\{a[1], \ldots, a[n]\}$ is a set of generators of $\mathcal{A}$. Let

$$
u(x[1], \ldots, x[m])=v(x[1], \ldots, x[m])
$$

be an identity.

To solve CHECK-ID $(\mathcal{A})$, we can either derive the identity or to prove its falsity. Therefore, we use a parallel run of two sequences of genetic algorithms.

Assumption 0: $\mathcal{A} \not \forall u(x[1], \ldots, x[m])=v(x[1], \ldots, x[m])$.

- A genetic algorithm GA[0,1] for selection a set $S$ of elements of $\mathcal{A}$.

- A genetic algorithm GA[0,2] for construction of homomorphism

$$
H: \mathcal{A} \rightarrow \mathcal{S}=\langle S \mid \Sigma\rangle
$$

where $\mathcal{S}$ is the subsemigroup of $\mathcal{A}$ generated by $S$.

- A genetic algorithm GA[0,3] for construction of multiplication table of $\mathcal{S}$.

- A genetic algorithm $\mathrm{GA}[0,4]$ for selection a set $F$ of elements of $\mathcal{S}$.

Consecutive run of genetic algorithms GA[0,1], GA[0,2], GA[0,3], GA[0,4] allows us to select values of $x[1], \ldots, x[m]$ that can potentially falsify the identity.

Assumption 1: $\mathcal{A} \models u(x[1], \ldots, x[m])=v(x[1], \ldots, x[m])$.

- A genetic algorithm $\mathrm{GA}[1,1]$ for selection a set of templates

$$
W=\left\{w \mid w \in(\{a[1], \ldots, a[n]\} \cup\{y[i] \mid i \in N\})^{+}\right\} .
$$


- A genetic algorithm GA[1,2] for discovery a set of equalities

$$
E=\{w[1]=w[2] \mid w[1], w[2] \in W, \mathcal{A}=w[1]=w[2]\}
$$

where we consider

$$
\{y[i] \mid i \in N\}
$$

as the set of variables.

- A genetic algorithm GA[1,3] for deduction a set of identities $T$.

- A genetic algorithm GA[1,4] for deduction

$$
u(x[1], \ldots, x[m])=v(x[1], \ldots, x[m]) .
$$

At first, we run GA[1,1] and create a set $W$. After this, we run GA[1,2]. GA[1,2] uses auxiliary genetic algorithm for initial prediction of elements of $E$. For initial value of $E$, we use a recursive parallel run of a genetic algorithm $\mathrm{GA}[1,4]$ for deduction of equalities and consecutive run of genetic algorithms GA $[0,1], \mathrm{GA}[0,2]$, GA[0,3], GA[0,4]. GA[1,4] allows us to prove some equalities from initial set $E$. Consecutive run of genetic algorithms GA[0,1], GA[0,2], GA $[0,3]$, GA $[0,4]$ we use to falsify some elements of $E$. GA[1,3] uses auxiliary genetic algorithm for initial prediction of elements of $T$. For initial value of $T$, we use a recursive run of GA[1,4] for deduction of identities. GA[1,4] uses four additional operators.

- Union of constants: if

$$
\mathcal{A} \models w[1](x)=w[2](x),
$$

for any value of a constant $x$, then we can consider $x$ as a variable.

- Separation of variables: if $w$ is a some word, $x$ is a variable, and $x \in w$, then we can replace $x$ by any element of $\mathcal{A}$.

- Multiplication: for any $w[1]$ and $w[2]$, we can consider $w[1] w[2]$.

- Substitution: for any variable $x$ and for any $w(x)$ and $u$, we can consider $w(u)$.

It is easy to see that we can use only GA[1,4] instead of usage of GA[1,1], GA[1,2], GA $[1,3]$, and GA[1,4]. Selected experimental results are given in Table 1.

In Table 1, we assume equal allocation of computing resources for the consideration of assumptions 0 and $1 . \mathrm{A}[1]$ is an algorithm that uses only GA[1,4] after $10^{5}$ generations. $\mathrm{A}[2]$ is an algorithm that uses $\mathrm{GA}[1,1], \mathrm{GA}[1,2], \mathrm{GA}[1,3]$, 


\begin{tabular}{|l|llll|}
\hline & $\mathrm{A}[1]$ & $\mathrm{A}[2]$ & $\mathrm{A}[3]$ & $\mathrm{A}[4]$ \\
\hline average time & $94.17 \mathrm{~h}$ & $72.89 \mathrm{~h}$ & $61.75 \mathrm{~h}$ & $42.3 \mathrm{~h}$ \\
\hline
\end{tabular}

Table 1: Experimental results for different genetic algorithms.

and $\mathrm{GA}[1,4]$ after $10^{3}$ generations. $\mathrm{A}[3]$ is an algorithm that uses $\mathrm{GA}[1,1]$, $\mathrm{GA}[1,2], \mathrm{GA}[1,3]$, and $\mathrm{GA}[1,4]$ after $10^{4}$ generations. $\mathrm{A}[4]$ is an algorithm that uses GA[1,1], GA[1,2], GA[1,3], and GA[1,4] after $10^{5}$ generations.

Since we use a parallel run of two sequences of genetic algorithms for the consideration of assumptions 0 and 1, we need some procedure to divide computing resources. In our computational experiments, we consider assumptions 0 and 1 on equal computing resources. Also, we consider a genetic algorithm for dynamic allocation of computing resources. Selected experimental results are given in Table 2.

\begin{tabular}{|l|lllll|}
\hline & ER & DA $\left(10^{3}\right)$ & $\mathrm{DA}\left(10^{4}\right)$ & $\mathrm{DA}\left(10^{5}\right)$ & $\mathrm{DA}\left(10^{6}\right)$ \\
\hline average time & $42.3 \mathrm{~h}$ & $39.57 \mathrm{~h}$ & $33.71 \mathrm{~h}$ & $11.84 \mathrm{~h}$ & $11.36 \mathrm{~h}$ \\
\hline
\end{tabular}

Table 2: Experimental results for resource allocation where ER denotes equal computing resources and $\mathrm{DA}(g)$ denotes dynamic allocation of computing resources with a genetic algorithm after $g$ generations.

ACKNOWLEDGEMENTS. The work was partially supported by Analytical Departmental Program "Developing the scientific potential of high school" 8.1616.2011.

\section{References}

[1] V.Yu. Popov, On the finite base property for semigroup varieties, Siberian Mathematical Journal, 43 (2002), 910-919.

[2] V.Yu. Popov, The property of having independent basis in semigroup varieties, Algebra and Logic, 44 (2005), 46-54.

[3] V.Yu. Popov, Equational theories for classes of finite semigroups, Algebra and Logic, 40 (2001), 55-66.

[4] V.Yu. Popov, Decidability of equational theories of coverings of semigroup varieties, Siberian Mathematical Journal, 42 (2001), 1132-1141.

[5] J. Almeida, M.V. Volkov, and S.V. Goldberg, Complexity of the identity checking problem for finite semigroups, Journal of Mathematical Sciences, 158 (2009), 605-614. 
[6] A. Gorbenko and V. Popov, On the Problem of Sensor Placement, Advanced Studies in Theoretical Physics, 6 (2012) 1117-1120.

[7] A. Gorbenko, M. Mornev, V. Popov, and A. Sheka, The problem of sensor placement for triangulation-based localisation, International Journal of Automation and Control, 5 (2011) 245-253.

[8] A. Gorbenko and V. Popov, Clustering Algorithm in Mobile Ad Hoc Networks, Advanced Studies in Theoretical Physics, 6 (2012) 1239-1242.

[9] A. Gorbenko and V. Popov, Multi-agent Path Planning, Applied Mathematical Sciences, 6 (2012) 6733-6737.

[10] A. Gorbenko and V. Popov, Programming for Modular Reconfigurable Robots, Programming and Computer Software, 38 (2012) 13-23.

[11] A. Gorbenko, M. Mornev, and V. Popov, Planning a Typical Working Day for Indoor Service Robots, IAENG International Journal of Computer Science, 38 (2011) 176-182.

[12] A. Gorbenko, V. Popov, and A. Sheka, Localization on Discrete Grid Graphs, Lecture Notes in Electrical Engineering, 107 (2012), 971-978.

[13] A. Gorbenko and V. Popov, The Problem of Selection of a Minimal Set of Visual Landmarks, Applied Mathematical Sciences, 6 (2012) 4729-4732.

[14] A. Gorbenko and V. Popov, On the Problem of Placement of Visual Landmarks, Applied Mathematical Sciences, 6 (2012) 689-696.

[15] A. Gorbenko and V. Popov, Footstep Planning for Humanoid Robots, Applied Mathematical Sciences, 6 (2012) 6567-6571.

[16] A. Gorbenko, A. Lutov, M. Mornev, and V. Popov, Algebras of Stepping Motor Programs, Applied Mathematical Sciences, 5 (2011), 1679-1692.

[17] V. Popov, Independent Systems of Semigroup Relations and Descriptions of Robotic Systems, Applied Mathematical Sciences, 6 (2012) 6725-6727.

[18] V. Popov, The word problem for relatively free semigroups, Semigroup Forum, 72 (2006), 1-14.

\section{Received: December 3, 2012}

